\title{
OPEN Neutrophil-to-lymphocyte ratio is associated with increased cerebral blood flow velocity in acute bacterial meningitis
}

Antje Giede-Jeppe ${ }^{1 凶}$, Selim Atay ${ }^{1}$, Julia Koehn ${ }^{1}$, Anne Mrochen ${ }^{1}$, Hannes Luecking ${ }^{2}$, Philip Hoelter ${ }^{2}$, Bastian Volbers ${ }^{1}$, Hagen B. Huttner ${ }^{3}$, Lena Hueske ${ }^{4,5}$ \& Tobias Bobinger $^{1,5}$

In community-acquired bacterial meningitis (CABM) intracranial vascular alterations are devastating complications which are triggered by neuroinflammation and result in worse clinical outcome. The Neutrophil-to-Lymphocyte ratio (NLR) represents a reliable parameter of the inflammatory response. In this study we analyzed the association between NLR and elevated cerebral blood flow velocity (CBFv) in CABM-patients. This study included all (CABM)-patients admitted to a German tertiary center between 2006 and 2016. Patients' demographics, in-hospital measures, neuroradiological data and clinical outcome were retrieved from institutional databases. CBFv was assessed by transcranial doppler (TCD). Patients', radiological and laboratory characteristics were compared between patients with/without elevated CBFv. Multivariate-analysis investigated parameters independently associated with elevated CBFv. Receiver operating characteristic(ROC-)curve analysis was undertaken to identify the best cut-off for NLR to discriminate between increased CBFv. 108 patients with CABM were identified. 27.8\% (30/108) showed elevated CBFv. Patients with elevated CBFv and normal CBFv, respectively had a worse clinical status on admission (Glasgow Coma Scale: 12 [9-14] vs. 14 [11-15]; $p=0.005)$ and required more often intensive care (30/30 [100.0\%] vs. 63/78 [80.8\%]; $p=0.01$ ). The causative pathogen was $S$. pneumoniae in $70 \%$. Patients with elevated CBFv developed more often cerebrovascular complications with delayed cerebral ischemia (DCI) within hospital stay $(p=0.031)$. A significantly higher admission-NLR was observed in patients with elevated CBFv (median [IOR]: elevated CBFv:24.0 [20.4-30.2] vs. normal CBFv:13.5 [8.4-19.5]; $p<0.001)$. Multivariate analysis, revealed NLR to be significantly associated with increased CBFv (Odds ratio $[95 \% \mathrm{CI}] 1.042$ [1.003-1.084]; $p=0.036$ ). ROC-analysis identified a NLR of 20.9 as best cut-off value to discriminate between elevated CBFv (AUC $=0.713, p<0.0001$, Youden's Index $=0.441$; elevated CBFv: NLR 20.9 19/30[63.5\%] vs. normal CBFv: NLR > 20.9 15/78[19.2\%]; $p<0.001$ ). Intracranial vascular complications are common among CABM-patients and are a risk factor for unfavorable outcome at discharge. Elevated NLR is independently associated with high CBFv and may be useful in predicting patients' prognosis.

Bacterial meningitis is a severe infection of the central nervous system ${ }^{1,2}$. In the development of bacterial meningitis the host inflammatory response plays an important role resulting in activation of both non cellular and cellular components of the immune system ${ }^{3,4}$. In the circulation neutrophils constitute the dominant cell type mediating the earliest innate immune responses getting to the site of infection ${ }^{5}$. Massive neutrophil recruitment is required across the blood-brain-barrier (BBB) to evoke a strong inflammatory response to react to the microorganisms at the bacterial infection site ${ }^{4}$. Activated immune cells within the brain, such as microglia, astrocytes and infiltrating leukocytes amplify the cascade of pro-inflammatory cytokines and cytotoxic agents. This activation of the immune response with subsequent rapid influx of leukocytes into the brain also causes adverse effects for the host as e.g. intracranial vascular alterations ${ }^{6}$. Intracranial vascular alterations may result

${ }^{1}$ Department of Neurology, University Hospital Erlangen, University of Erlangen-Nuremberg, Schwabachanlage 6, 91054 Erlangen, Germany. ${ }^{2}$ Department of Neuroradiology, University of Erlangen-Nuremberg, Erlangen, Germany. ${ }^{3}$ Department of Neurology, University Hospital Gießen, Gießen, Germany. ${ }^{4}$ Neurological Hospital for Parkinson's Disease, Beelitz-Heilstaetten, Germany. ${ }^{5}$ These authors contributed equally: Lena Hueske and Tobias Bobinger. ${ }^{\square}$ email: Antje.Giede-Jeppe@uk-erlangen.de 


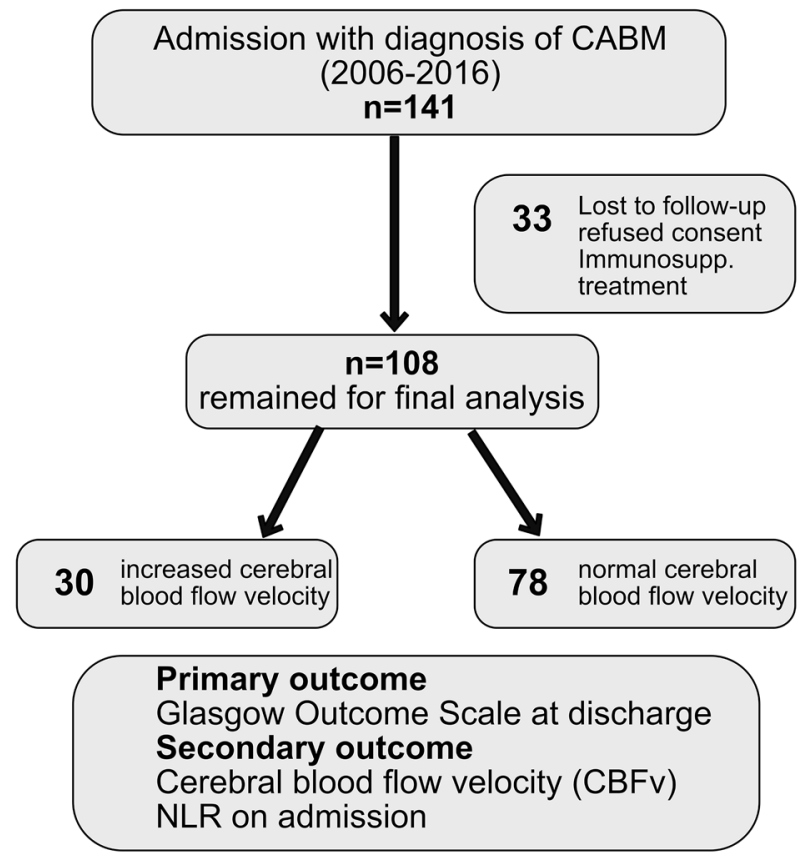

Figure 1. Flowchart of patients. 141 patients with community-acquired bacterial meningitis were identified during the study period. After exclusion of 33 patients 108 patients remained for further analysis. Patients were dichotomized according to increased cerebral blood flow velocity $(\mathrm{N}=30)$ and normal cerebral blood flow velocity $(\mathrm{N}=78)$. Abbreviations: $C A B M$ community acquired bacterial meningitis, $C B F v$ cerebral blood flow velocity, GOS Glasgow outcome scale (range, 5 no or mild deficit, to 1, death), NLR Neutrophil-to-Lymphocyte ratio.

in damage to cortical and subcortical structures ${ }^{7,8}$ which further result in edema, hydrocephalus and increased intracranial pressure ${ }^{9}$. Therefore they represent a devastating complication leading to unfavorable outcome and increased morbidity ${ }^{10}$. The inflammatory process in bacterial meningitis can be monitored by numerous biochemical markers ${ }^{11,12}$. The Neutrophil-to-Lymphocyte ratio (NLR) represents information on both the innate and adaptive immune system and is a reliable parameter for the general immune response to various stimuli. Neutrophil-to-Lymphocyte ratio (NLR), calculated by absolute neutrophil count divided by absolute lymphocyte count, is easy to perform in routine practice and cost-effective. Neutrophil-to-Lymphocyte ratio (NLR) has proven its prognostic value in cerebrovascular ${ }^{13,14}$ and inflammatory diseases ${ }^{15}$, several types of tumors ${ }^{16-18}$, infections ${ }^{19,20}$ and also in the differential diagnosis between viral and bacterial meningitis ${ }^{21,22}$. This study aimed to analyze the association between NLR and elevated cerebral blood flow velocity (CBFv) which represents a devastating complication in bacterial meningitis.

\section{Methods}

Patients und Inclusion criteria. All consecutive patients with diagnosis of CABM admitted to the Department of Neurology, University Hospital Erlangen, Germany were included in a prospective longitudinal institutional database, which was approved by the institutional ethics committee. Out of this database, all patients $(\mathrm{N}=141)$ admitted between 2006 and 2016 for treatment of CABM have been recruited to this study. We excluded patients receiving permanent immunomodulatory treatment (e.g. corticosteroids, methotrexate, cytostatic drugs and biologicals) on admission $(n=14)$. Patients without follow-up data or refused consent were also excluded $(\mathrm{n}=19) .108$ patients remained for final analysis (Fig. 1).

Data collection. Data of all patients were retrieved from our institutional prospective database: patients' history (hypertension, diabetes mellitus, alcohol abuse or other comorbidities), GCS (Glasgow Coma Scale) on admission, clinical symptoms on admission, laboratory findings on admission (cerebrospinal fluid and blood work, causative pathogen), clinical course, outcome and neurologic findings at discharge, and treatment.

Arterial vascular alterations and incidence of cerebral ischemia were assessed using computed tomography imaging (CT-scan, (SIEMENS Somatom Volume Zoom, Somatom Sensation 64, Somatom Definition AS+; Siemens Healthcare, Forchheim, Germany) or magnetic resonance imaging (MRI) (SIEMENS Magnetom Sonata 1.5T, Magnetom Aera 1.5T, Siemens Healthcare, Erlangen, Germany).

NLR was calculated by dividing the absolute neutrophil count by the absolute lymphocyte count (NLR days $1,2,3,4,5,6,7,8)$. 
Diagnosis of acute bacterial meningitis (ABM). The diagnosis of acute bacterial meningitis was confirmed by identification of the causative pathogen in cerebrospinal fluid and/or blood via microscopic Gram stain, CSF/blood cultured isolates and PCR on $\mathrm{CSF}^{23}$. In case the causative pathogen could not be identified bacterial meningitis was diagnosed by typical CSF findings as mainly granulocytic CSF white blood cell count greater than $1000 \mathrm{cells} / \mu \mathrm{l}$, an increase of CSF protein of more than $100 \mathrm{mg} / \mathrm{dl}$, and a CSF/serum glucose ratio less than 0.3 and/or presence of clinical symptoms as fever, neck stiffness, headache, impaired consciousness ${ }^{24}$. CSF lactate level was measured using standard enzymatic test.

Detection of increased cerebral blood flow velocity. Presence of vascular alterations was assessed by TCD as a routine clinical practice termed as cerebral blood flow velocity (CBFv) of the anterior cerebral artery (ACA), middle cerebral artery (MCA), internal cerebral artery (ICA), posterior cerebral artery (PCA), and the basilar artery (BA). Systolic CBFv greater than $150 \mathrm{~cm} / \mathrm{s}_{\text {sere considered increased }}{ }^{25,26}$.

Outcome. Outcome at discharge was evaluated according to the Glasgow Outcome Scale (1-5) by two physicians, trained and certified for data collection: A score of 1 on this scale indicates death; a score of 2, a vegetative state; a score of 3, severe disability (the patient is not able to live independently but can follow commands); a score of 4, moderate disability (the patient is capable of living independently but unable to return to work); and a score of 5 , mild or no disability (the patient is able to return to work). A favorable outcome was defined as a score of 5, and an unfavorable outcome as a score of 1-4. The Glasgow Outcome Scale is a well-validated instrument with high interobserver agreement ${ }^{27,28}$. Long-term outcome was evaluated using the modified Rankin Scale (mRS) 3 and 12 months after onset. Favorable outcome representing an independent clinical status was defined as mRS score of $0-2$, unfavorable outcome as mRS score of $3-6^{29}$.

Statistical analysis. Statistical analysis was performed using SPSS 22.0 (IBM Analytics, Armonk, New York, United States) and GraphPad Prism 8 (GraphPad Software, San Diego, California, United States). Categorial variables were presented as frequency and percentage, Pearson chi square and Fisher's exact test were used to compare between these groups. For continuous variables, the Kolmogorov-Smirnov test was used to test the distribution of data. If data showed normal distribution, data was presented with mean $\pm S D$ and the Student $t$ test was used for analysis. Data lacking normal distribution, median and interquartile range were shown and the Mann-Whitney U test was used for comparison. Significance level was set at $\alpha=0.05$. All parameters showing a statistical trend $(p<0.1)$ were included in a multivariate model to identify parameters independently associated with elevated cerebral blood flow. A Receiver Operating Characteristic (ROC) curve and Youden's J statistic was used to determine the cut-off value for $\mathrm{NLR}^{30}$. Then, patients were dichotomized according to the identified cut-off value.

Ethics approval and consent to participate. All procedures involving human participants were in accordance with the ethical standards of the institutional research committee of the University of ErlangenNuremberg and with the 1964 Helsinki declaration and its later amendments. The Institutional Ethics Committee of the University of Erlangen-Nuremberg had approved the study protocol (304_16 B).

Informed consent. Informed consent was obtained from all subjects.

\section{Results}

Patient characteristics. Overall 108 patients with CABM remained for final analysis (Fig. 1). The overall cohort of patients was $59 \pm 16$ years old. $51.9 \%(56 / 108)$ were male. More than half of the patients presented with clinical signs suggestive of bacterial meningitis as fever $57.4 \%(62 / 108)$, headache $51.9 \%(56 / 108)$ and meningism $63.9 \%$ (69/108). Only $27.8 \%$ (30/108) of these patients showed further neurological deficits. $74.1 \%(80 / 108)$ received dexamethasone on admission. In $18 / 108$ patients $(16.7 \%)$ no pathogen was detected (Supplementary Table 1).

Vascular complications. $\quad 30 / 108$ (27.8\%) patients developed elevated cerebral blood flow velocity within 4 (3-5) days after admission. Patients with elevated CBFv versus normal CBFv: had a worse clinical status on admission (median [IQR]: Glasgow Coma Scale 12 [9-14] vs. 14 [11-15]; $p=0.005$, neurologic deficit 13/30 [43.3\%] vs. $17 / 78$ [21.8\%]; $p=0.025)$, required more often intensive care $(30 / 30$ [100\%] vs. $63 / 78$ [80.8\%]; $p=0.010)$ with need of osmotherapy $(13 / 30[43.3 \%]$ vs. $6 / 78[7.7 \%] ; p<0.001)$, therapy with nimodipine $(23 / 30$ [76.7\%] vs. $3 / 78[3.9 \%] ; p<0.001)$ and/or catecholamines $(22 / 30[73.3 \%]$ vs. $31 / 78[39.7 \%] ; p=0.002)$ at a dedicated intensive care unit. They needed longer ventilation ( $167 \mathrm{~h}[45-510]$ vs. $0 \mathrm{~h}[0-206] ; p=0.001)$ because of reduced consciousness. In patients with increased CBFv CSF analysis confirmed typical abnormalities as polymorphonuclear leukocytosis, decreased glucose concentration, and increased protein concentration. Whereas only protein concentrations were significantly increased $(2.910 \mathrm{~g} / \mathrm{L}$ [1.491-4.287] vs. $1.501 \mathrm{~g} / \mathrm{L}$ [0.629-3.038]; $p=0.009)$. The most common causative microorganism was Streptococcus pneumoniae (70\% [21/30] vs. $26.9 \%$ $[21 / 78] ; p<0.001)$ identified by CSF $(28 / 30$ [93.3\%] vs. $51 / 78$ [65.4\%]; $p=0.003)$. In $3.3 \%(1 / 30(3.3 \%)$ vs. 17/78 $(21.8 \%), p=0.021)$ no causative pathogen was identified. These patients showed a higher rate of infectious complications (sepsis: $17 / 30$ [56.7\%] vs. 30/78 [38.5\%]; $p=0.087$ ). Cerebral infarctions during hospital stay occurred more frequently $(9 / 27[33.3 \%]$ vs. $3 / 30[10.0 \%] ; p=0.031)$ translating into an unfavorable outcome at discharge (Glasgow Outcome Scale $3[3,4]$ vs. $4[3-5] ; p=0.028$, Table 1). Infarcts morphologically due to cerebral vasos- 


\begin{tabular}{|c|c|c|c|}
\hline Meningitis & $\begin{array}{l}\text { Increased cerebral blood flow } \\
\text { velocity }(\mathrm{N}=30)\end{array}$ & $\begin{array}{l}\text { Normal cerebral blood flow } \\
\text { velocity }(\mathrm{N}=78)\end{array}$ & $p$ value \\
\hline Gender $(+)^{\mathrm{b}}$ & $23(76.7 \%)$ & $33(42.3 \%)$ & 0.001 \\
\hline \multicolumn{4}{|c|}{\begin{tabular}{|l|} 
Prior medical history-admission status-in hospital measures \\
\end{tabular}} \\
\hline Premorbid mRS ${ }^{\mathrm{a}}$ & $0(0-1)$ & $0(0-1)$ & 0.278 \\
\hline Alcohol abuse $^{\mathrm{b}}$ & $9(30.0 \%)$ & $6(7.7 \%)$ & 0.003 \\
\hline Neurologic deficit ${ }^{\mathrm{b}}$ & $13(43.3 \%)$ & $17(21.8 \%)$ & 0.025 \\
\hline Glasgow Coma Scale $^{\mathrm{a}}$ & $12(9-14)$ & $14(11-15)$ & 0.005 \\
\hline Length of ventilation (LOV) (h) ${ }^{\mathrm{a}}$ & $167(45-510)$ & $0(0-206)$ & 0.001 \\
\hline Dexamethasone on admission $^{\mathrm{b}}$ & $26(86.7 \%)$ & $54(69.2 \%)$ & 0.064 \\
\hline Stay on Neurointensive Care Unit ${ }^{\mathrm{b}}$ & $30(100.0 \%)$ & $63(80.8 \%)$ & 0.010 \\
\hline Osmotherapy $^{\mathrm{b}}$ & $13(43.3 \%)$ & $6(7.7 \%)$ & $<0.001$ \\
\hline Nimodipine therapy $^{\mathrm{b}}$ & $23(76.7 \%)$ & $3(3.9 \%)$ & $<0.001$ \\
\hline Need of Catecholamines $^{\mathrm{b}}$ & $22(73.3 \%)$ & $31(39.7 \%)$ & 0.002 \\
\hline Temperature on admission $\left({ }^{\circ} \mathrm{C}\right)$ & $38.3(37.5-39.2)$ & $38.8(38.1-39.4)$ & 0.064 \\
\hline Sepsis $^{\mathrm{b}}$ & $17(56.7 \%)$ & $30(38.5 \%)$ & 0.087 \\
\hline \multicolumn{4}{|l|}{\begin{tabular}{|l|} 
Laboratory values on admission \\
\end{tabular}} \\
\hline \multicolumn{4}{|l|}{ First spinal tap } \\
\hline Leucocytes $\left(\times 10^{6} / \mathrm{L}\right)^{\mathrm{a}}$ & $2328(187-5788)$ & $1020(216-5104)$ & 0.661 \\
\hline Erythrocytes $\left(\times 10^{6} / \mathrm{L}\right)^{\mathrm{a}}$ & $234(5-555)$ & $27(1-160)$ & 0.032 \\
\hline Proteine $(\mathrm{g} / \mathrm{L})^{\mathrm{a}}$ & $2.910(1.491-4.287)$ & $1.501(0.629-3.038)$ & 0.009 \\
\hline Glucose $(\mathrm{mmol} / \mathrm{L})^{\mathrm{a}}$ & $1.28(0-3.61)$ & $2.28(0.22-3.44)$ & 0.676 \\
\hline Lactate $(\mathrm{mmol} / \mathrm{L})^{\mathrm{a}}$ & $9.8(5.2-16.9)$ & $7.1(3.5-13.8)$ & 0.178 \\
\hline Causative pathogen identified by blood ${ }^{\mathrm{b}}$ & $17(56.7 \%)$ & $31(39.7 \%)$ & 0.113 \\
\hline Causative pathogen identified by CSF $^{b}$ & $28(93.3 \%)$ & $51(65.4 \%)$ & 0.003 \\
\hline Causative pathogen identified by CSF via $\mathrm{PCR}^{\mathrm{b}}$ & $14(46.7 \%)$ & $28(35.9 \%)$ & 0.304 \\
\hline No causative pathogen identified ${ }^{\mathrm{b}}$ & $1(3.3 \%)$ & $17(21.8 \%)$ & $\mid 0.021$ \\
\hline \multicolumn{4}{|l|}{ Most frequent causative pathogen } \\
\hline S. pneumoniae ${ }^{\mathrm{b}}$ & $21(70.0 \%)$ & $21(26.9 \%)$ & $<0.001$ \\
\hline \multicolumn{4}{|l|}{ Serum } \\
\hline Neutrophil-Lymphocyte-Ratio ${ }^{a}$ & $24.0(20.4-30.2)$ & $13.5(8.4-19.5)$ & $<0.001$ \\
\hline Thrombocytes $\left(10^{9} / \mathrm{L}\right)^{c}$ & $172.7 \pm 62$ & $208.0 \pm 98$ & 0.078 \\
\hline Granulocytes $\left(10^{9} / \mathrm{L}\right)^{\mathrm{a}}$ & $15.6(13.7-20.8)$ & $12.1(8.1-15.4)$ & 0.017 \\
\hline Lymphocytes $\left(10^{9} / \mathrm{L}\right)^{\mathrm{a}}$ & $0.7(0.6-1.0)$ & $0.9(0.8-1.3)$ & 0.024 \\
\hline \multicolumn{4}{|l|}{ Radiological data } \\
\hline \multicolumn{4}{|l|}{ First CT on admission } \\
\hline Abscess $^{\mathrm{b}}$ & $1(3.3 \%)$ & $2(2.6 \%)$ & 0.627 \\
\hline Ischemia $^{\mathrm{b}}$ & $2(6.7 \%)$ & $2(2.6 \%)$ & 0.308 \\
\hline Obstructive hydrocephalus $^{\mathrm{b}}$ & $4(13.3 \%)$ & $1(1.3 \%)$ & 0.020 \\
\hline \multicolumn{4}{|c|}{ CT within hospital stay- follow up (57/108 patients received a second cerebral imaging) } \\
\hline Abscess $^{\mathrm{b}}$ & $3 / 27(11.1 \%)$ & $3 / 30(10.0 \%)$ & 0.613 \\
\hline Ischemia $^{\mathrm{b}}$ & 9/27 (33.3\%) & $3 / 30(10.0 \%)$ & 0.031 \\
\hline Obstructive hydrocephalus $^{\mathrm{b}}$ & $3 / 27(11.1 \%)$ & $1 / 30(3.3 \%)$ & 0.266 \\
\hline \multicolumn{4}{|l|}{ Discharge status } \\
\hline Symptomatic epilepsyb & $6(20.0 \%)$ & $3(3.8 \%)$ & 0.013 \\
\hline Glasgow Outcome Scale (GOS) & $3(3-4)$ & $4(3-5)$ & 0.028 \\
\hline $\mathrm{LOV}^{\mathrm{b}}$ & $167(45-510)$ & $0(0-206)$ & 0.001 \\
\hline Length of stay $(\mathrm{d})^{\mathrm{a}}$ & $22(18-26)$ & $15(9-20)$ & $<0.001$ \\
\hline mRS at discharge ${ }^{a}$ & $4(2-5)$ & $1(1-4)$ & \begin{tabular}{|l|}
0.020 \\
\end{tabular} \\
\hline mRS at 3 months ${ }^{a}$ & $2(2-4)$ & $2(1-3)$ & 0.166 \\
\hline mRS at 12 months ${ }^{a}$ & $2(1-4)$ & $1(0-3)$ & 0.238 \\
\hline
\end{tabular}

Table 1. Baseline characteristics, laboratory data, in-hospital measures and outcome parameters for all patients with community-acquired bacterial meningitis developing increased cerebral blood flow velocity. ${ }^{\mathrm{a}}$ Median (IQR), ${ }^{\mathrm{b}} \mathrm{No} .(\%),{ }^{\mathrm{c} M e a n} \pm$ standard deviation. Abbreviations: CBFv cerebral blood flow velocity, GOS Glasgow outcome scale (range, 5 no or mild deficit, to 1, death), $m R S$ modified Rankin Scale (range 0 , no deficit, to 6, death), NIHSS Scale National Institutes of Health Stroke Scale, NLR Neutrophil-to-Lymphocyte ratio, $I Q R$ interquartile range, CSF Cerebrospinal fluid. $p$-value: Boldface type indicates statictical significance, findings showing a statistical trend are expressed in italics. 


\begin{tabular}{|l|l|l|}
\hline Parameters & $\mathbf{9 5 \%}$ CI & $\boldsymbol{p}$ value \\
\hline NLR D1 & $1.042(1.003-1.084)$ & $\mathbf{0 . 0 3 6}$ \\
\hline Catecholamine therapy & $0.634(0.137-2.944)$ & 0.561 \\
\hline Osmotherapy & $0.181(0.040-0.819)$ & $\mathbf{0 . 0 2 6}$ \\
\hline Neurologic deficit on admission & $2.693(0.784-9.254)$ & 0.116 \\
\hline Ventilation & $3.001(0.606-14.857)$ & 0.178 \\
\hline
\end{tabular}

Table 2. Multivariate analysis of parameters associated with increased cerebral blood flow velocity. Multivariable regression analysis was calculated for the association with increased cerebral blood flow velocity. Only parameters showing a statistical trend $(p<0.1)$ in prior univariate testing were included in the multivariable model. For each parameter risk ratio and $95 \%$ confidence interval are provided. Significant findings are expressed in bold. NLR Neutrophil-to-Lymphocyte ratio, 95\% CI Confidence Interval.

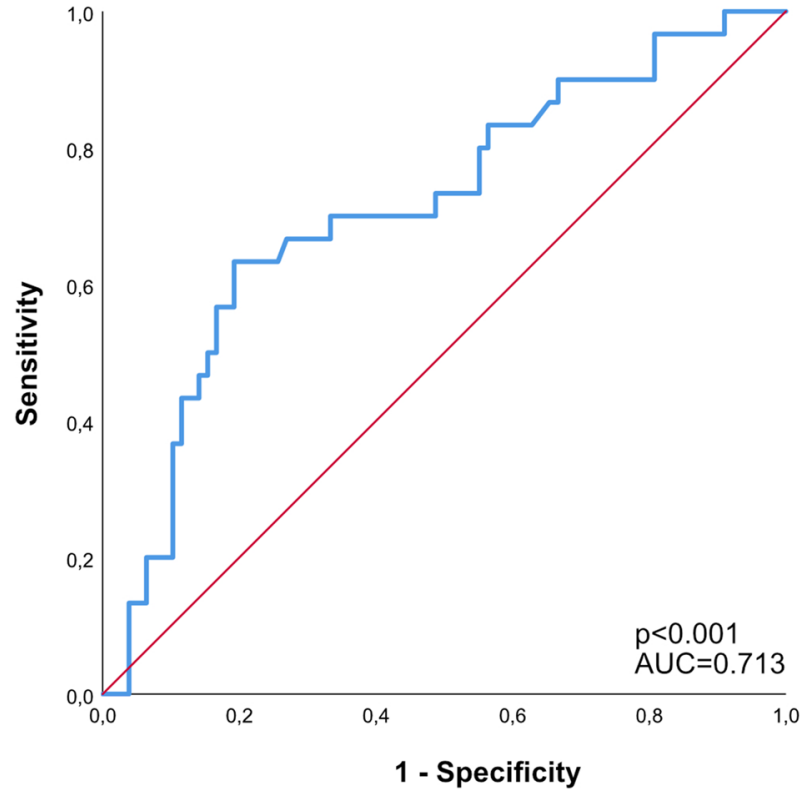

Figure 2. Association of Neutrophil-to-Lymphocyte ratio (NLR) with increased cerebral blood flow velocity. Receiver operating characteristic (ROC) - curve for prediction of increased cerebral blood flow velocity. ROC plot demonstrated the AUC for increased cerebral blood flow velocity (AUC $=0.713 ; p<0.001$, Youden's index $=0.441$; sensitivity, $63.3 \%$; specificity, $80.8 \%$ ). The cut-off value was detected at 20.9 .

pasm were predominantly located in the vascular territory of the anterior and/or middle cerebral artery (Supplementary Table 2).

Further, a significantly higher NLR on admission was observed in patients with elevated blood flow velocity (median [IQR]: elevated CBFv: 24.0 [20.4-30.2] vs. normal CBFv: 13.5 [8.4-19.5]; $p<0.001$ ). Parameters with tendency for significance $(p<0.1)$ in univariate testing were included into a multivariable model. In this model NLR levels on admission were associated with increased cerebral blood flow velocity (1.042 (1.003-1.084); $p=0.036$; Table 2) as well as need of osmotherapy $(p=0.026)$. Need of catecholamine therapy $(p=0.561)$, clinical status on admission $(p=0.116)$ and need of ventilation $(p=0.178)$ were not significantly associated with increased CBFv.

Association of Neutrophil-to-Lymphocyte ratio with increased cerebral blood flow velocity. ROC-analysis identified a NLR of 20.9 as the best cut-off threshold on admission to discriminate between increased $\mathrm{CBFv}$ within hospital stay $(\mathrm{AUC}=0.713 ; p<0.001$, Youden's index $=0.441$; sensitivity, $63.3 \%$; specificity, 80.8\%; elevated CBFv: NLR $\geq 20.9$ 19/30[63.3\%] vs. NLR $<20.915 / 78$ [19.2\%]; $p<0.001$; Fig. 2). These patients showed an unfavorable outcome (GOS 1-4) at discharge (elevated CBFv 27/30 [90.0\%] vs. normal CBFv 51/78 [65.4\%]; $p=0.01$, Fig. 3). 


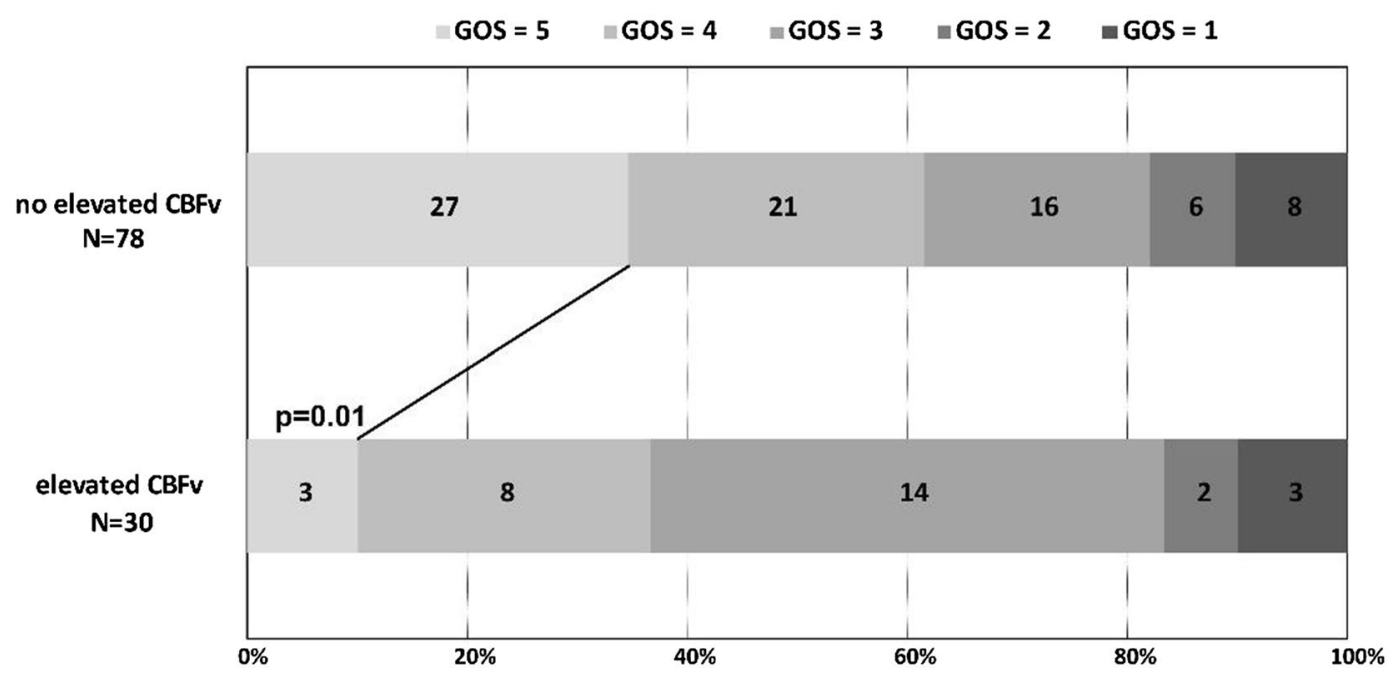

Figure 3. Glasgow Outcome Scale at discharge with elevated CBF versus no elevated CBF. Illustration of the proportion of patients with elevated cerebral blood flow velocity $(n=30)$ and no/normal elevated cerebral blood flow velocity $(\mathrm{n}=78)$. Favorable functional outcome was defined as $\mathrm{GOS}=5$, unfavorable functional outcome as GOS $=1-4$. $P$ values were calculated for the comparison of unfavorable functional outcome among patients elevated and normal CBFv $(p=0.01)$. Abbreviations: $C B F v$ cerebral blood flow velocity, GOS Glasgow outcome scale (range, 5 no or mild deficit, to 1 , death).

\section{Discussion}

There are three major findings from this cohort study on CABM-patients: (1) We identified elevated NLR on admission to be associated with increased CBFv within hospital stay. (2) ROC-analysis revealed a NLR of 20.9 as the best cut-off threshold on admission to discriminate between increased CBFv within hospital stay. (3) These patients showed an unfavorable outcome (GOS 1-4) at discharge.

According to published data ${ }^{21,22}$ NLR represents a promising parameter in the differential diagnosis of acute bacterial meningitis predominantly in patients aged 15 and older. As this dataset does not provide CSF counts on lymphocytes and neutrophils we are unable to reproduce these findings.

Cerebral vasospasms represent a devastating complication in acute bacterial meningitis contributing to an unfavorable functional outcome $\mathrm{e}^{10,31}$. Our results imply that patients with an increased NLR on admission may yield a potential risk to develop an elevated $\mathrm{CBFv}$ which contributes to a potentially severe course of disease. Of 108 patients $30(27.8 \%)$ developed increased cerebral blood flow velocity within hospital stay due to infection with S. pneumoniae in $70 \%$ in agreement of published data ${ }^{31,32}$. Among these patients ischemic complications were observed in $21.1 \%$. TCD was performed routinely whereas cerebral imaging was performed according to clinical signs and symptoms. As not every patient with increased cerebral blood flow velocity underwent CT/ MRI-imaging subclinical strokes may remained undetected. Further, increased flow velocities do not necessarily cause impending ischemic complications ${ }^{26}$.

In our cohort of patients with community acquired bacterial meningitis we identified a predictive NLR-cut off level to be $\geq 20.9$. This cut-off value is increased compared to published data for e.g. prediction of ICH after endovascular thrombectomy in acute ischemic stroke ${ }^{33}$.

Unexpectedly and contrary to published data ${ }^{34,35}$ we did not identify dexamethasone administration to be independently associated with increased cerebral blood flow velocity in multivariate analysis. Prior to the wide spread use of adjunctive steroids in bacterial meningitis ${ }^{36,37}$ cerebral vascular alterations were devastating but common complications. Reasons may be cerebral vasculitis, septic emboli, intraarterial thrombosis or disseminated intravascular coagulation ${ }^{35,38-40}$. Thus specific anti-inflammatory regimes are desirable ${ }^{41,42}$ beyond adjuvant corticosteroid treatment which has been proven beneficial on case fatality rates in adult patients with pneumococcal meningitis but only in high-income countries ${ }^{37}$.

In acute intracerebral haemorrhage ${ }^{14}$, subarachnoid haemorrhage ${ }^{43}$ and ischemic stroke ${ }^{44}$ elevated NLRlevels were linked to unfavorable functional outcome. This could be shown by our data only in univariate testing (GOS 3 [3,4] vs. 4 [3-5]; p=0.028, mRS 4 [2-5] vs. 1 [1-4]; $p=0.020$ ). Regarding long-term-outcome we did not identify significant differences. Reasons for this finding may be the small cohort size. According to recently published data, NLR represents a promising marker in stroke for the prediction of symptomatic hemorrhagic transformation (sHT) undergoing revascularization ${ }^{45}$ and cerebral edema after reperfusion therapy ${ }^{46}$.

Taken together, increased cerebral blood flow velocity is associated with neuroinflammation and represents a devastating complication in bacterial meningitis. NLR is an easy accessible and cost-effective biomarker that has proven its prognostic value in several conditions among cerebrovascular ${ }^{14,47}$ as well as infectious diseases ${ }^{19,48}$ and also in the differential diagnosis between viral and bacterial meningitis ${ }^{21,22}$. As a reliable parameter for the general immune response to various stimuli, NLR does not represent an inflammatory marker within a specific entity. Therefore, it remains unclear whether increased NLR-levels on admission reflect the state of bacterial infections or if high NLR values represent an independent surrogate to predict a severe course of the disease 
with intracranial vascular alterations ${ }^{34}$ as a devastating complication in bacterial meningitis. Future studies should investigate whether elevated NLR-levels in CABM-patients help to identify patients suitable for immune modulating treatments.

Our study has several limitations. First of all the small cohort size comprising only 108 patients weakens our data. It lacks a prospective and multi-center design. Except for admission laboratory data were not available every day within hospital stay. Bloodwork and CSF stains were done as required by clinical practice and not by scheduled timing. Further assessment of $\mathrm{CBFv}$ was conducted by different examiners within the inclusion period. As critical care procedures improved within the last years this may impose bias to the reported data. Finally, above findings do not provide any mechanistic evidence confirmed by specific blood sampling analyses or detailed immunoprofiling, and therefore depict a phenomenological finding.

\section{Conclusions}

Among CABM-patients intracranial vascular complications are a risk factor for unfavorable outcome at discharge. Elevated NLR is independently associated with high CBFv and may be useful in predicting patients' prognosis. Future studies should investigate whether the impact of NLR represents an independent clinical implication or a preexisting comorbidity.

\section{Data availability}

All data generated or analysed during this study are included in this published article.

Received: 2 December 2020; Accepted: 17 May 2021

Published online: 31 May 2021

\section{References}

1. McIntyre, P. B., O’Brien, K. L., Greenwood, B. \& van de Beek, D. Effect of vaccines on bacterial meningitis worldwide. Lancet 380(9854), 1703-1711 (2012).

2. van de Beek, D. Progress and challenges in bacterial meningitis. Lancet 380(9854), 1623-1624 (2012).

3. Kasanmoentalib, E. S. et al. Complement factor H contributes to mortality in humans and mice with bacterial meningitis. J. Neuroinflamm. 16(1), 279 (2019).

4. Mohanty, T. et al. Neutrophil extracellular traps in the central nervous system hinder bacterial clearance during pneumococcal meningitis. Nat. Commun. 10(1), 1667 (2019).

5. Nauseef, W. M. \& Borregaard, N. Neutrophils at work. Nat. Immunol. 15(7), 602-611 (2014).

6. Eisenhut, M. Vasospasm in cerebral inflammation. Int. J. Inflamm. 2014, 509707 (2014).

7. Mook-Kanamori, B. B., Geldhoff, M., van der Poll, T. \& van de Beek, D. Pathogenesis and pathophysiology of pneumococcal meningitis. Clin. Microbiol. Rev. 24(3), 557-591 (2011).

8. Engelen-Lee, J. Y., Brouwer, M. C., Aronica, E. \& van de Beek, D. Pneumococcal meningitis: clinical-pathological correlations (MeninGene-Path). Acta Neuropathol. Commun. 4, 26 (2016).

9. Petersdorf, R. G., Swarner, D. R. \& Garcia, M. Studies on the pathogenesis of meningitis. II. Development of meningitis during pneumococcal bacteremia. J. Clin. Investig. 41, 320-327 (1962).

10. Pfister, H. W., Borasio, G. D., Dirnagl, U., Bauer, M. \& Einhaupl, K. M. Cerebrovascular complications of bacterial meningitis in adults. Neurology 42(8), 1497-1504 (1992).

11. Thorsdottir, S., Henriques-Normark, B. \& Iovino, F. The role of microglia in bacterial meningitis: inflammatory response, experimental models and new neuroprotective therapeutic strategies. Front. Microbiol. 10, 576 (2019).

12. Geldhoff, M. et al. Inflammasome activation mediates inflammation and outcome in humans and mice with pneumococcal meningitis. BMC Infect. Dis. 13, 358 (2013).

13. Giede-Jeppe, A. et al. Increased neutrophil-to-lymphocyte ratio is associated with unfavorable functional outcome in acute ischemic stroke. Neurocrit. Care 33, 97-104 (2019).

14. Lattanzi, S., Cagnetti, C., Provinciali, L. \& Silvestrini, M. Neutrophil-to-lymphocyte ratio predicts the outcome of acute intracerebral hemorrhage. Stroke 47(6), 1654-1657 (2016).

15. Boulos, D. et al. The neutrophil-lymphocyte ratio in early rheumatoid arthritis and its ability to predict subsequent failure of triple therapy. Semin. Arthritis Rheum. 49(3), 373-376 (2019).

16. Patel, D. A. et al. Neutrophil-to-lymphocyte ratio as a predictor of survival in patients with triple-negative breast cancer. Breast Cancer Res. Treat. 174(2), 443-452 (2019).

17. Lalani, A. A. et al. Change in neutrophil-to-lymphocyte ratio (NLR) in response to immune checkpoint blockade for metastatic renal cell carcinoma. J. Immunother. Cancer 6(1), 5 (2018).

18. Capone, M. et al. Baseline neutrophil-to-lymphocyte ratio (NLR) and derived NLR could predict overall survival in patients with advanced melanoma treated with nivolumab. J. Immunother. Cancer 6(1), 74 (2018).

19. Pantzaris, N. D., Platanaki, C., Pierrako, C., Karamouzos, V. \& Velissaris, D. Neutrophil-to-lymphocyte ratio relation to sepsis severity scores and inflammatory biomarkers in patients with community-acquired pneumonia: a case series. J. Transl. Intern. Med. 6(1), 43-46 (2018).

20. Cataudella, E. et al. Neutrophil-to-lymphocyte ratio: an emerging marker predicting prognosis in elderly adults with communityacquired pneumonia. J. Am. Geriatr. Soc. 65(8), 1796-1801 (2017).

21. Mentis, A. A., Kyprianou, M. A. \& Tzanakaki, G. Age-specific application of neutrophil-to-lymphocyte ratio in meningitis: a nationwide study. Eur. J. Clin. Microbiol. Infect. Dis. 36(9), 1553-1557 (2017).

22. Mentis, A. F., Kyprianou, M. A., Xirogianni, A., Kesanopoulos, K. \& Tzanakaki, G. Neutrophil-to-lymphocyte ratio in the differential diagnosis of acute bacterial meningitis. Eur. J. Clin. Microbiol. Infect. Dis. 35(3), 397-403 (2016).

23. van de Beek, D. et al. ESCMID guideline: diagnosis and treatment of acute bacterial meningitis. Clin. Microbiol. Infect. Off. Publ. Eur. Soc. Clin. Microbiol. Infect. Dis. 22(Suppl 3), S37-62 (2016).

24. van de Beek, D. et al. Clinical features and prognostic factors in adults with bacterial meningitis. N. Engl. J. Med. 351(18), 1849-1859 (2004).

25. Klein, M. et al. Arterial cerebrovascular complications in 94 adults with acute bacterial meningitis. Crit. Care 15(6), R281 (2011).

26. Laumer, R. et al. Cerebral hemodynamics in subarachnoid hemorrhage evaluated by transcranial Doppler sonography. Part 1. Reliability of flow velocities in clinical management. Neurosurgery 33(1), 1-8 (1993).

27. Jennett, B. \& Bond, M. Assessment of outcome after severe brain damage. Lancet 1(7905), 480-484 (1975).

28. McMillan, T. et al. The Glasgow Outcome Scale: 40 years of application and refinement. Nat. Rev. Neurol. 12(8), 477-485 (2016).

29. Rankin, J. Cerebral vascular accidents in patients over the age of 60. I. General considerations. Scott. Med. J. 2(4), 127-136 (1957). 
30. Hanley, J. A. \& McNeil, B. J. The meaning and use of the area under a receiver operating characteristic (ROC) curve. Radiology 143(1), 29-36 (1982).

31. Pfister, H. W., Feiden, W. \& Einhaupl, K. M. Spectrum of complications during bacterial meningitis in adults. Results of a prospective clinical study. Arch. Neurol. 50(6), 575-581 (1993).

32. Haring, H. P. et al. Time course of cerebral blood flow velocity in central nervous system infections. A transcranial Doppler sonography study. Arch. Neurol. 50(1), 98-101 (1993).

33. Pikija, S. et al. Neutrophil to lymphocyte ratio predicts intracranial hemorrhage after endovascular thrombectomy in acute ischemic stroke. J. Neuroinflamm. 15(1), 319 (2018).

34. Gallegos, C., Tobolowsky, F., Nigo, M. \& Hasbun, R. Delayed cerebral injury in adults with bacterial meningitis: a novel complication of adjunctive steroids?. Crit. Care Med. 46(8), e811-e814 (2018).

35. Lucas, M. J., Brouwer, M. C. \& van de Beek, D. Delayed cerebral thrombosis in bacterial meningitis: a prospective cohort study. Intensive Care Med. 39(5), 866-871 (2013).

36. de Gans, J. \& van de Beek, D. Dexamethasone in adults with bacterial meningitis. N. Engl. J. Med. 347(20), 1549-1556 (2002)

37. Brouwer, M. C., McIntyre, P., Prasad, K. \& van de Beek, D. Corticosteroids for acute bacterial meningitis. Cochrane Database Syst. Rev. 9, CD004405 (2015).

38. Khedher, A. et al. Cerebral vasculitis complicating pneumococcal meningitis. Eur. J. Case Rep. Intern. Med. 5(5), 000819 (2018).

39. Lucas, M. J., Brouwer, M. C., van der Ende, A. \& van de Beek, D. Endocarditis in adults with bacterial meningitis. Circulation 127(20), 2056-2062 (2013).

40. Kastenbauer, S. \& Pfister, H. W. Pneumococcal meningitis in adults: spectrum of complications and prognostic factors in a series of 87 cases. Brain J. Neurol. 126(Pt 5), 1015-1025 (2003).

41. Kasanmoentalib, E. S., Valls Seron, M., Morgan, B. P., Brouwer, M. C. \& van de Beek, D. Adjuvant treatment with dexamethasone plus anti-C5 antibodies improves outcome of experimental pneumococcal meningitis: a randomized controlled trial. J. Neuroinflamm. 12, 149 (2015).

42. Sprong, T. et al. Inhibition of C5a-induced inflammation with preserved C5b-9-mediated bactericidal activity in a human whole blood model of meningococcal sepsis. Blood 102(10), 3702-3710 (2003).

43. Tao, C. et al. Clinical value of neutrophil to lymphocyte and platelet to lymphocyte ratio after aneurysmal subarachnoid hemorrhage. Neurocrit. Care 26(3), 393-401 (2017).

44. Gokhan, S. et al. Neutrophil lymphocyte ratios in stroke subtypes and transient ischemic attack. Eur. Rev. Med. Pharmacol. Sci. 17(5), 653-657 (2013).

45. Switonska, M. et al. Neutrophil-to-lymphocyte ratio and symptomatic hemorrhagic transformation in ischemic stroke patients undergoing revascularization. Brain Sci. 10(11), 771 (2020).

46. Ferro, D. et al. Neutrophil-to-lymphocyte ratio predicts cerebral edema and clinical worsening early after reperfusion therapy in stroke. Stroke 52(3), 859-867 (2021).

47. Liesz, A. et al. Stress mediators and immune dysfunction in patients with acute cerebrovascular diseases. PLoS ONE 8(9), e74839 (2013).

48. Nam, K. W. et al. High neutrophil-to-lymphocyte ratio predicts stroke-associated pneumonia. Stroke 49, 1886-1892 (2018).

\section{Author contributions}

A.G.J.: Design and conceptualized the study; analyzed the data; drafted the manuscript for intellectual content. S.A.: Conception and design of the study, acquisition of data. J.K.: Acquisition of data, interpretation of data, critical review of manuscript. A.M.: Acquisition of data, interpretation of data, critical review of manuscript. H.L.: Acquisition of radiological data, interpretation of data, critical review of manuscript. P.H.: Acquisition of radiological data, interpretation of data, critical review of manuscript. B.V.: Conception and design of the study, statistical analysis of the data, critical review of the manuscript. H.B.H.: Conception and design of the study, interpretation of the data, critical review of the Manuscript. L.H.: Conception and design of the study, acquisition of data, critical review of the manuscript. T.B.: Conception and design of the study Interpretation of the data; revised the manuscript for intellectual content.

\section{Funding}

Open Access funding enabled and organized by Projekt DEAL.

\section{Competing interests}

Dr. Giede-Jeppe reports no disclosures. Mr. Atay reports no disclosures. Dr. Koehn reports no disclosures. Dr. Mrochen reports no disclosures. Dr. Luecking reports no disclosures. Dr. Hoelter reports no disclosures. Dr. Volbers reports personal fees from Pfizer AG/Bristol-Myers Squibb SA, personal fees from Bayer AG, grants from Institutional grant (Inselspital), personal fees from Ipsen Pharma, personal fees from CSL Behring, outside the submitted work. Prof. Huttner reports research grants by Novartis, Medtronic, UCB Pharma and Portola Pharmaceuticals. HBH reports personal fees from Bayer AG, Boehringer Ingelheim, Daiichi Sankyo, Medtronic, CLS Behring, UCB Pharma and Portola Pharmaceuticals. Dr. Hueske reports no disclosures. Dr. Bobinger reports no disclosures.

\section{Additional information}

Supplementary Information The online version contains supplementary material available at https://doi.org/ 10.1038/s41598-021-90816-0.

Correspondence and requests for materials should be addressed to A.G.-J.

Reprints and permissions information is available at www.nature.com/reprints.

Publisher's note Springer Nature remains neutral with regard to jurisdictional claims in published maps and institutional affiliations. 
(c) (i) Open Access This article is licensed under a Creative Commons Attribution 4.0 International cc) License, which permits use, sharing, adaptation, distribution and reproduction in any medium or format, as long as you give appropriate credit to the original author(s) and the source, provide a link to the Creative Commons licence, and indicate if changes were made. The images or other third party material in this article are included in the article's Creative Commons licence, unless indicated otherwise in a credit line to the material. If material is not included in the article's Creative Commons licence and your intended use is not permitted by statutory regulation or exceeds the permitted use, you will need to obtain permission directly from the copyright holder. To view a copy of this licence, visit http://creativecommons.org/licenses/by/4.0/.

(C) The Author(s) 2021 